\title{
Brain Resting State Functional Networks in Infants with Prenatal Opioid Exposure
}

Nicole Steinhardt ${ }^{1,2}$, , Ramana Vishnubhotla ${ }^{1}$, Yi Zhao ${ }^{3}$, David Haas ${ }^{4}$, Gregory Sokol, $^{5}$, Senthilkumar Sadhasivam ${ }^{6}$ Rupa Radhakrishnan ${ }^{1,2}$

${ }^{1}$ Department of Radiology and Imaging Sciences, Indiana University School of Medicine, Indianapolis, IN, USA; ${ }^{2}$ Indiana University School of Medicine, Indianapolis, IN, USA;

${ }^{3}$ Department of Biostatistics, Indiana University School of Medicine, Indianapolis, IN, USA

${ }^{4}$ Department of Obstetrics and Gynecology, Indiana University School of Medicine,

Indianapolis, IN, USA ; ${ }^{5}$ Department of Pediatrics, Indiana University School of Medicine, Indianapolis, IN, USA; ${ }^{6}$ Department of Anesthesiology, Indiana University School of Medicine, Indianapolis, IN, USA

\section{Purpose:}

Infants of mothers with opioid and substance use can present with postnatal withdrawal symptoms and are at risk of poor neurodevelopmental outcomes in later childhood. Identifying methods to evaluate the consequences of substance exposure on the developing brain can help initiate proactive therapies to improve outcomes for opioid-exposed neonates. Additionally, early brain imaging in infancy has the potential to identify early brain developmental alterations that could prognosticate neurodevelopmental outcomes in these children. In this study, we aim to identify differences in global brain network connectivity in infants with prenatal opioid exposure compared to healthy control infants, using resting-state functional MRI performed at less than 2 months completed gestational age.

Materials and Methods:

In this prospective, IRB-approved study, we recruited 20 infants with prenatal opioid exposure and 20 healthy, opioid naïve infants. Anatomic imaging and resting-state functional MRI were performed at less than 48 weeks corrected gestational age, and rs-fMRI images were coregistered to the UNC neonate brain template and 90 anatomic atlas-labelled regions. Covariate Assisted Principal (CAP) regression was performed to identify brain network functional connectivity that was significantly different among infants with prenatal opioid exposure compared to healthy neonates.

Results:

Of the 5 significantly different CAP components identified, the most distinct component (CAP5, $p=3.86 \times 10^{-6}$ ) spanned several brain regions, including the right inferior temporal gyrus, bilateral Hesch's gyrus, left thalamus, left supramarginal gyrus, left inferior parietal lobule, left superior parietal gyrus, right anterior cingulate gyrus, right gyrus rectus, left supplementary motor area, and left pars triangularis. Functional connectivity in this network was lower in the infants with prenatal opioid exposure compared to non-opioid exposed infants.

\section{Conclusion:}

This study demonstrates global network alterations in infants with prenatal opioid exposure compared to non-opioid exposed infants. Future studies should be aimed at identifying clinical significance of this altered connectivity. 